\title{
REPOSTAJE EN TIERRA DE UN AVIÓN MEDIANTE ALGORITMOS GENÉTICOS
}

\author{
Elías Plaza \\ eliasplaza@gmail.com \\ Matilde Santos \\ Facultad de Informática, Universidad Complutense de Madrid, msantos@ucm.es
}

\begin{abstract}
Resumen
En este trabajo se presenta una solución alternativa, basada en técnicas de la Inteligencia Artificial, al problema de la gestión de la distribución de combustible dentro de una aeronave comercial. En concreto se ha abordado el repostaje de la aeronave antes de un vuelo. El repostaje en tierra consiste en decidir la cantidad de combustible que se dispondrá en cada depósito atendiendo a criterios de seguridad, estabilidad del avión y eficiencia energética. Para ello se define un problema de optimización cuya función de coste nace del conocimiento especifico de los factores que influyen en una aeronave que tiene relación con el posicionamiento del combustible. Después su resolución se realiza mediante Algoritmos Genéticos. Los resultados obtenidos en simulación mejoran la solución manual estándar que se viene aplicando.
\end{abstract}

Palabras Clave: Algoritmos Genéticos, optimización, repostaje de combustible, arquitectura de los depósitos, aeronave comercial.

\section{INTRODUCCIÓN}

En este trabajo se plantea el sistema de control para gestionar la distribución de combustible entre los tanques de un avión comercial real tipo Long Range (por ejemplo un Airbus A330 [1]).

Este tipo de aviones consta de varios depósitos de combustible situados en sus alas (del orden de seis tanques), más un depósito situado en la cola que sirve para balancear el peso del avión y así tener más control sobre el centrado del mismo. Esto tiene gran influencia en la estabilidad del avión así como en su controlabilidad. Por tanto, es necesario disponer de un sistema de control de la distribución de combustible entre los tanques para mantener el centro de gravedad dentro de unos márgenes de seguridad adecuados. Además dicho sistema debe gestionar el combustible de tal forma que los motores siempre queden suministrados correctamente.
Debido a su complejidad, la estrategia de redistribución abarca dos fases. En primer lugar el repostaje de la aeronave antes de un vuelo, y en segundo lugar, la redistribución del combustible entre los tanques durante el vuelo de la aeronave.

El primero de los problemas, el de repostaje en tierra, consiste en decidir la cantidad de combustible que se dispondrá en cada depósito. Para ello se define un problema de optimización cuya función de coste nace del conocimiento específico de los factores que influyen en una aeronave que tiene relación con el posicionamiento del combustible. En este trabajo se presenta una propuesta basada en la técnica heurística evolutiva de los Algoritmos Genéticos para su resolución [7], y se compara con la aproximación manual estándar.

Aunque la literatura sobre este tema es escasa, en el artículo de Turgut [8] se utilizan algoritmos genéticos para analizar la relación entre el consumo de fuel de una aeronave y la altitud de la misma durante la fase de descenso. También hace referencia a un problema similar en el sector aeronáutico el trabajo de Mongeau [5] sobre la optimización de la distribución de la carga de pago en un avión. Sin embargo hay que apuntar que en este caso el problema es discreto ya que el tamaño de contenedor es fijo; además el algoritmo busca maximizar la carga para cada vuelo de entre toda la mercancía a transportar. En nuestro caso la masa de fuel es fija para un vuelo dado, y el problema es continuo ya que la cantidad de combustible en cada tanque estará comprendida entre cero y el máximo que puede acoger el tanque.

La organización de esta contribución es como sigue. En la sección 2 se presentan las especificaciones de la aeronave. En la sección 3 se describe la configuración del algoritmo genético utilizado y la función de coste que define el problema a optimizar. La sección 4 muestra la aplicación al problema concreto y los resultados son presentados y comentados en la sección 5. El trabajo termina con las conclusiones y trabajos futuros. 


\section{DESCRIPCIÓN DEL PROBLEMA}

Como es sabido, justo antes de despegar la aeronave debe repostar. La cantidad de combustible que se debe cargar se determina a partir del plan de vuelo concreto aprobado por la autoridad. Para un determinado plan le corresponderá una carga mínima obligatoria. Básicamente esta carga, además de tener en cuenta la cantidad de combustible que previsiblemente consumirán los motores (más un margen de seguridad), también contiene, o bien la cantidad de combustible necesaria para mantenerse a la espera en hipódromo durante un determinado tiempo antes de tener permiso para aterrizar en el destino, o bien la cantidad necesaria para aterrizar en el aeropuerto alternativo si no es posible hacerlo en el de destino [3].

Tomando como base la arquitectura típica del sistema de combustible de un avión comercial de largo alcance (como el A330-200), se tienen dos depósitos en cada ala, un depósito central en la panza y un depósito en la cola de la aeronave, todos ellos intercomunicados por tuberías (un total de 6 tanques). La distribución de combustible se controla por válvulas y bombas.

Los depósitos 2 y 4 (ver [6]) se denominan Inner, ya que se encuentran en la parte más interior de cada semi-ala. Los depósitos 1 y 5 son llamados Outer, situándose estos en una zona más alejada del encastre de cada ala. Los depósitos 1 y 2 son simétricos respecto a 4 y 5 , a cada lado del plano vertical de simetría del avión. Además, debido a la forma del ala y por conveniencia de la distribución inercial, los depósitos Inner son mucho más grandes en capacidad que los Outer. El depósito 3 es el denominado Central, por estar situado en la panza de la aeronave entre los encastres de las alas. Su capacidad es del mismo orden de magnitud que los Inner. Por su parte el depósito 6 es conocido como Trim Tank ya que está dispuesto en el interior del estabilizador horizontal de trimado. Su capacidad es bastante menor al Central aunque algo mayor que los Outer.

Esta tarea de decisión sobre el repostaje se encuentra muy reglada para cada aeronave particular. La cantidad de combustible total a repostar va a ser un dato de entrada para nuestro problema.

Otro dato de entrada es el peso total de la aeronave. A partir de él se calcula el centro de gravedad (CG) objetivo que conviene tener en el eje longitudinal del avión para su maniobrabilidad $\mathrm{y}$ el margen de estabilidad, definido por la posición longitudinal máxima y mínima que puede alcanzar el centro de gravedad. Estos tres valores (CG objetivo, $\mathrm{CG}$ máximo, CG mínimo) se pueden obtener de funciones que provee el fabricante del avión. Las usadas en este caso se presentan en las Figuras 1 y 2, generadas con magnitudes de aviones reales [4].

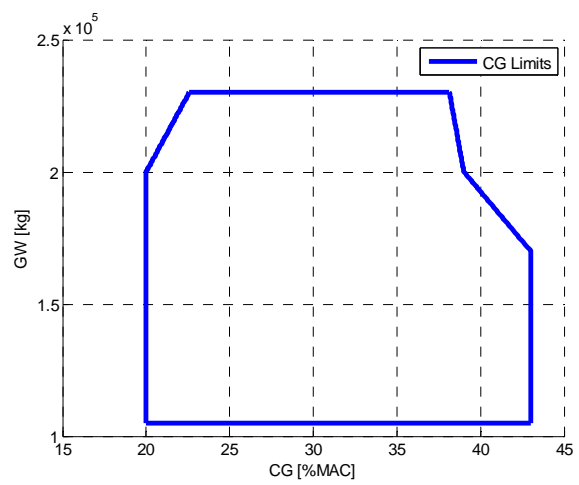

Figura 1: Límites del centro de gravedad en función de la masa total.

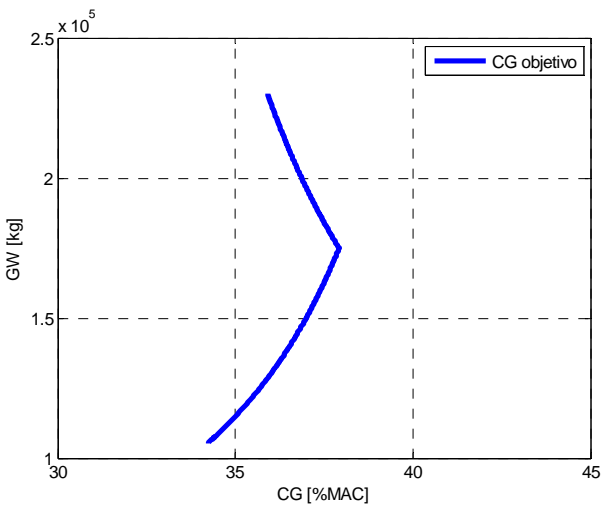

Figura 2: Centro de gravedad objetivo en función de la masa total.

El peso total de la aeronave GW (Gross Weight) viene dado por tres componentes independientes. El primero es fijo: el peso del avión en vacío EW (Empty Weight), dado por todo el conjunto estructural de la aeronave así como la carga de sistemas. Los otros dos son variables. Uno de ellos es la carga de pago del avión MP (Mass Payload), formada por tripulación, pasaje, equipaje $\mathrm{y}$ mercancía. La suma de la carga de EW más MP da lugar al peso sin fuel de la aeronave, ZFW (Zero Fuel Weight). El tercer conjunto es la cantidad de combustible, MF (Mass Fuel).

$$
\begin{aligned}
& G W(k g)=Z F W(k g)+M F(k g) \\
& Z F W(k g)=E W(k g)+M P(k g)
\end{aligned}
$$

Además diferenciamos la masa prevista para consumo, porque no toda la masa cargada se consumirá; siempre se llevan reservas que permitan llegar a un aeropuerto alternativo en caso de emergencia o mantenerse en hipódromo. Esta masa será de interés por su influencia en la seguridad operacional así que será otro dato de entrada más a nuestro problema. 
La operación de repostaje consiste en distribuir la cantidad total de fuel entre los tanques. Cada compañía elige una forma de llevar a cabo esta operación durante la fase de pre-vuelo. Este orden está estudiado para mantener un llenado lógico y que mantenga el CG dentro de la envolvente si la carga de pago también se ha colocado correctamente. Sin embargo no optimiza la distribución. La distribución manual que habitualmente se utiliza, además de poder incurrir en errores humanos, resulta más lenta que si la automatizamos con alguna técnica como los algoritmos evolutivos. Además el problema de colocación de la carga de pago pasa a ser menos exigente, ya que cierto error en la colocación de la carga puede suplirse con la distribución del fuel.

\section{RESTRICCIONES Y FUNCIÓN DE COSTE}

El objetivo de la optimización es distribuir la carga de fuel de un avión entre los tanques haciendo que el centro de gravedad quede dentro de los límites de estabilidad y lo más cerca posible del CG objetivo. Para ello se va a definir una función de coste a minimizar. Los componentes o costes de esta función vienen dados por los siguientes criterios:

- Coste 1: la distancia entre el centro de gravedad resultante y el objetivo debe ser mínima (2).

$$
C 1=\left|X_{c g_{-} o b j}-X_{c g}\right|+\left|Y_{c g_{-} o b j}-Y_{c g}\right|+\left|Z_{c g_{-} o b j}-Z_{c g}\right|_{(2)}
$$

Donde el CG objetivo viene determinado por la masa total (ZFW+MF), y el CG de la aeronave se calcula aplicando la ley de masas (3).

$$
\overrightarrow{C G}=\frac{Z F W \cdot \overrightarrow{C G}_{z f w}+\sum_{i=1}^{6}\left(\overrightarrow{C G}_{d e p_{-} i} \cdot M_{i}\right)}{Z F W+\sum_{i=1}^{6}\left(M_{i}\right)}
$$

con $\mathrm{CG}_{\text {dep_i }}$ la posición del centro de gravedad y $\mathrm{M}_{\mathrm{i}}$ la masa de cada depósito de combustible.

- Coste 2: la diferencia entre la cantidad de combustible cargada en los tanques que abastecen los motores y el combustible que previsiblemente consumirán durante el vuelo. Esto tiene como objetivo la seguridad operacional y minimizar la cantidad de fuel que habrá que redistribuir en vuelo, lo que reduce el gasto energético de las bombas (4).

$$
C 2=\left(M_{\text {eng } 1} / 2-M_{2}+M_{\text {eng } 2} / 2-M_{4}\right) / M F
$$

Donde $\mathrm{M}_{\text {eng }}$ es la masa prevista para el consumo de los motores $(\mathrm{kg}), \mathrm{M}_{2}$ es la masa de combustible en el tanque "2" (kg) y $\mathrm{M}_{4}$ la del "4" (kg), la de los Inner.
- Coste 3: la inversa de la diferencia entre la cantidad de fuel cargada en cada depósito frente a la crítica. Se trata de minimizar el desplazamiento del CG durante el despegue debido a la inercia que tiene el líquido a ir hacia atrás por la aceleración. Para cada depósito existe una cantidad de combustible para la cual el desplazamiento de CG es máximo dada una aceleración, cantidad que llamaremos masa crítica.

Para construir este coste se ha recurrido a una función definida a tramos para cada tanque. Dicha función vale "1" para el rango de masas en que el desplazamiento es máximo durante la operación de despegue. Fuera de ese rango se interpola hasta el valor de la función "0" en los puntos en que el desplazamiento es mínimo.

Dependiendo del avión las curvas del desplazamiento del centro de gravedad respecto a la carga de fuel en cada tanque es distinto. En la Figura 3 se presentan los correspondientes a órdenes de magnitud de aviones como el considerado en este trabajo.

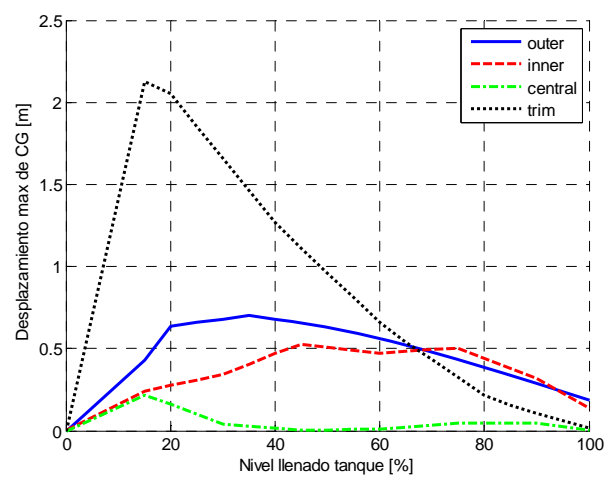

Figura 3: Desplazamiento máximo del CG para un porcentaje de llenado durante un despegue típico.

En base a esta gráfica se obtienen las funciones que dan lugar a este coste, con $\mu=M_{i} / M_{\text {max }_{-} i} \cdot 100$, porcentaje de llenado de cada tanque. A modo de ejemplo se muestra en (5) la del tanque central.

$$
\begin{gathered}
f_{\text {central }}=\left\{\begin{array}{cc}
1, & \text { si }\{\mu>10 \| \mu<20\} \\
1-\frac{10-\mu}{10}, & \text { si }\{\mu \leq 10\} \\
1+\frac{20-\mu}{80}, & \text { si }\{\mu \geq 20\}
\end{array}\right. \\
C 3=f_{\text {central }}+2 \cdot f_{\text {inner }}+2 \cdot f_{\text {outer }}+f_{\text {trim }}
\end{gathered}
$$

- Coste 4: la cantidad de fuel cargada en los depósitos que no tienen tomas de repostaje (tanques internos). Se trata de minimizar el tiempo de repostaje, y por tanto el tiempo que el avión debe permanecer en tierra. Se debe tener en cuenta la cantidad que ya está cargada en el avión inicialmente (6).

$$
C 4=\left(100 M_{1}+M_{2}+50 M_{3}+M_{4}+100 M_{5}+1000 M_{6}\right) / M F
$$


- Coste 5: la diferencia entre las cantidades de combustibles cargadas en cada depósito para realizar la distribución lo más homogénea posible y así repartir el peso a lo largo del ala. Aunque no es crítico, a la larga disminuiría la fatiga del ala.

$$
C 5=\left(\left|M_{1}-M_{2}\right|+\left|M_{4}-M_{5}\right|\right) / M F
$$

La función de coste vendrá definida por la suma de esos costes, que dependen de las masas de los depósitos y datos de entrada al problema, multiplicados cada uno por un peso según su prioridad. La sintonía de esos costes se basa en la experiencia acerca del problema.

$$
\begin{aligned}
& C\left(Z F W, M_{\text {eng }}, M_{i}\right)=P_{1} \cdot C 1\left(Z F W, M_{i}\right) \\
& +P_{2} \cdot C 2\left(M_{\text {eng }}, M_{i}\right)+P_{3} \cdot C 3\left(M_{i}\right)+P_{4} \cdot C 4\left(M_{i}\right)+P_{5} \cdot C 5\left(M_{i}\right)
\end{aligned}
$$

Es importante observar que algunos de los costes siguen criterios contrarios entre ellos. Por ejemplo, el coste C2 obtiene su mínimo cuando la masa de fuel en los depósitos Inner iguala a la prevista para el consumo en el vuelo. Sin embargo, el coste C5 es mínimo cuando las masas Inner y Outer son iguales (hay que recordar que la masa máxima posible en los Outer es muy baja). Como las soluciones pueden ser múltiples, se buscan estos pesos para llegar a la mejor solución de compromiso basada en la experiencia. De esta manera se ha decidido dar más peso a $\mathrm{C} 1$ y $\mathrm{C} 2$ frente a los demás, y el peso más pequeño a $\mathrm{C} 5$.

La minimización de la función de coste está supeditada a las siguientes restricciones:

- Restricción 1: no superar el volumen máximo de cada depósito.

$$
R 1: M_{i} \leq M_{\text {max }_{-} i}
$$

- Restricción 2: no permitir masas negativas.

$$
R 2: M_{i} \geq 0.0
$$

- Restricción 3: no salir del rango válido del centro de gravedad impuesto (11).

$$
R 3: X_{C G \min }(Z F W+M F) \leq X_{C G} \leq X_{C G \max }(Z F W+M F)
$$

- Restricción 4: mínima cantidad de combustible obligatoria.

$$
R 4: \sum_{i=1}^{6} M_{i} \geq M F
$$

- Restricción 5: dejar espacio libre suficiente en el Trim Tank para retrasar el CG durante el vuelo, siendo $\eta$ un porcentaje de la masa máxima de este tanque prefijado en base a la experiencia sobre el retraso del CG necesario durante un vuelo.

$$
R 5: M_{6} \leq \eta \cdot M_{\text {max }_{-} 6}
$$

\section{APLICACIÓN DEL ALGORITMO GENÉTICO}

El repostaje en tierra tiene una doble vertiente: la resolución del problema de optimización y la sintonización de la función objetivo (los pesos).

El vector de decisión es la cantidad de fuel que hay que introducir en cada depósito. Lo más directo es elegir la masa como magnitud de esta cantidad. Tenemos seis depósitos, así que tendremos un vector de decisión de seis componentes ordenadas según el número que identifica cada depósito.

$$
v_{\text {decision }}=\left[\begin{array}{llll}
M 1 & \text { M2 M3 M4 M5 M6 }
\end{array}\right.
$$

Dado que la suma de las cantidades de cada depósito debe ser la masa total de fuel que se ha determinado para la operación de la aeronave, la sexta componente es la diferencia entre la masa total (dato) y la suma del resto de componentes. Hemos decidido que esta componente sea el tanque central (M3) por ser el que tiene mayor tamaño y está más centrado en el avión.

$$
V_{\text {individuo }}=\left[\begin{array}{lll}
\text { M1 M2 M4 M5 M6 }] & \text { M }
\end{array}\right.
$$

Las entradas son: masa total de fuel, masa total de la aeronave (para obtener los límites de CG y el CG objetivo), y la masa de fuel prevista para el consumo.

Respecto al algoritmo genético, aunque la configuración la puede determinar el usuario, hemos considerado una población inicial de $\mathrm{N}=30$ individuos, de los cuales tomamos $\mathrm{Np}=8$ parejas de padres en cada iteración. Para generar la población inicial hemos considerado varias opciones, por ejemplo, generar de manera aleatoria cada componente del vector individuo, pero puede generar soluciones no factibles. Otra opción es el llenado secuencial de los depósitos, repartiendo la masa total en cascada, y después perturbar cada individuo sumándole una cantidad aleatoria entre 0 y el $10 \%$ de la masa máxima del tanque en cuestión. La mayor parte de los individuos son factibles, cumpliendo todas las restricciones, pero la perturbación asimétrica desplaza el CG hacia la izquierda o derecha, algo no deseable. Por lo tanto, se optó por aplicar esta última opción pero con una perturbación simétrica (sumamos lo mismo a los tanques que se encuentran a un lado y al otro del ala).

\subsection{OPERADORES GENÉTICOS}

Los padres son seleccionados de entre todos los individuos siguiendo el método de la ruleta.

Aunque se han considerado tres versiones del cruce (asimétrico, binario simulado y cruce binario con restricciones), pero se ha optado por la primera por 
ser la más sencilla ya que no se obtenía una mejora significativa con las otras tras varios experimentos.

Para la mutación de hijos obtenidos en el cruce hemos considerado mutación uniforme, mutación semi-aleatoria sobre un número aleatorio de componentes (se suma a las componentes a mutar un valor comprendido entre 0 y el $20 \%$ de la masa máxima del tanque), y si de forma aleatoria una componente del vector es seleccionada para ser mutada, también se muta el tanque simétrico, que ha resultado la elegida por dar mejores resultados.

En la población de partida se sustituyen los padres elegidos por los hijos resultantes del cruce y mutación de los primeros.

Como criterio de parada se ha establecido un máximo de 5000 iteraciones que son asumibles en tiempo de computación y la convergencia es buena. También se tiene en cuenta para parar la ejecución si la aptitud del mejor individuo de la población no mejora durante 100 iteraciones consecutivas.

\section{VALIDACIÓN Y RESULTADOS}

El algoritmo diseñado es codificable directamente dentro de la computadora FCMS (Sistema de Control y Monitorización de Fuel) del avión. Los datos necesarios son de dos tipos: fijos, correspondientes a la geometría del avión, y los variables, correspondientes a los parámetros de entrada (ZFW, $\mathrm{CG}, \mathrm{M}_{\mathrm{eng}}$ ), que provienen de la conexión digital ARINC-429 de otra computadora (el FMGC, encargada de la gestión del plan de vuelo). La solución del problema son las masas de combustible en cada tanque. Una vez se tienen estos valores, el FCMC se encarga de abrir las válvulas de paso de cada tanque y bombear el combustible a cada tanque hasta alcanzar dichas masas.

El tiempo de resolución no puede ser mayor de 5 minutos ya que la fase de pre-vuelo se puede ver afectada por retrasos.

\subsection{ANÁLISIS DE LA EVOLUCIÓN}

Partimos de una solución lejana a los óptimos y además no factible, por ejemplo:

$$
\left.v_{\text {individo }}\right|_{\text {inic }}[2865,0,0,2865,40000]
$$

Hemos considerado en este escenario que la aeronave repostará $75000 \mathrm{~kg}$ de combustible, con una carga de pago de $10000 \mathrm{~kg}$, situándose el CG con ZFW a 0.4 $\mathrm{m}$ por detrás del punto de referencia, borde de ataque del MAC (cuerda media aerodinámica). Con esto, el vector de decisión inicial sería:

$$
v_{\text {decision }\left.\right|_{\text {inic }}}=\left[\begin{array}{c}
2865 \\
0 \\
75000-2865-2865-40000 \\
0 \\
2865 \\
40000
\end{array}\right]=\left[\begin{array}{c}
2865 \\
0 \\
29270 \\
0 \\
2865 \\
40000
\end{array}\right]
$$

Es decir, no cargamos los tanques Inner, cargamos los tanques Outer al máximo, el Central casi al completo también, y el Trim Tank muy por encima de su capacidad. Evidentemente esta solución no es factible (R1). Además la posición del CG también está fuera del margen de estabilidad, situándose muy por detrás del límite situado para la configuración de este escenario ( $7 \mathrm{~m}$ del origen).

Aplicando el algoritmo, la evolución de los costes para el mejor individuo de la población en cada iteración se muestra en la Figura 4. Observamos que el algoritmo enseguida tiende a generar soluciones factibles y a empezar a disminuir los costes, sobre todo de aquellos que más peso tienen en la función criterio como es la posición del CG. Por otra parte, se observa también como una vez encontrada una solución bastante aceptable (a partir de la iteración 200 aproximadamente), ya es muy difícil mejorar al individuo encontrado.

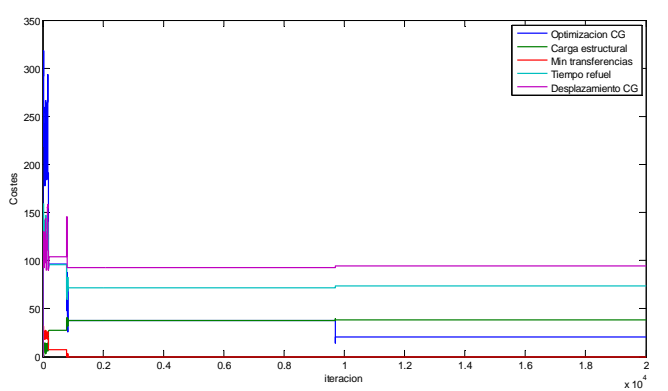

Figura 4: Evolución de los costes para cada variable.

La Figura 5 muestra la evolución de la aptitud máxima de la población en cada iteración. En las primeras iteraciones es nula, debido a que dichos individuos no son solución factible. En el momento que se encuentra un individuo factible se produce un salto en la aptitud. A partir de aquí el algoritmo trata de encontrar una mejor mediante cruce y mutación.

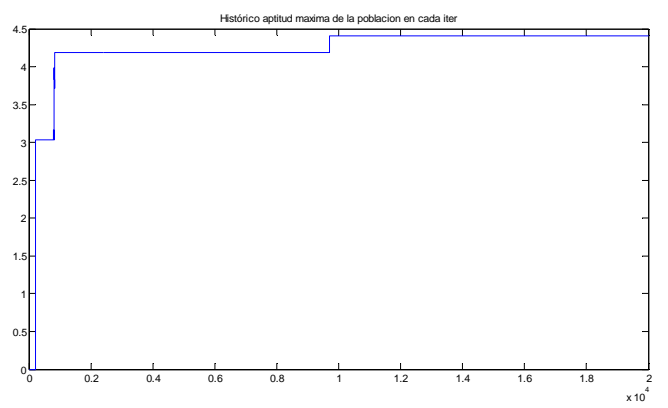

Figura 5: Aptitud del mejor individuo. 
Es evidente que en nuestro diseño prima la optimización de la posición longitudinal del CG, lo que se hace evidente si presentamos la evolución del CG del mejor individuo en cada iteración (Figura 6).

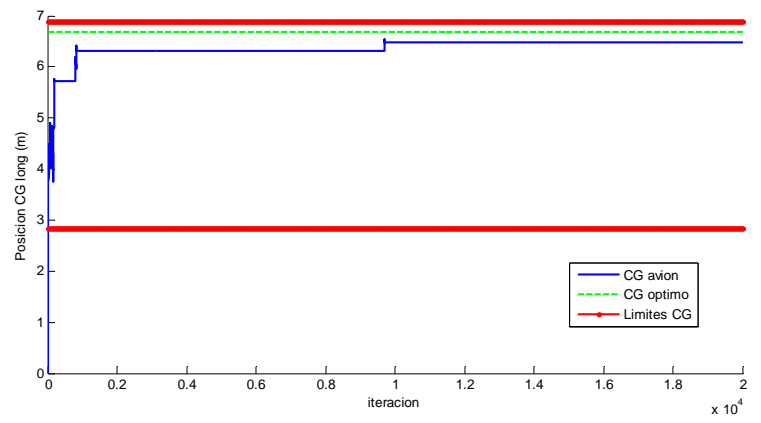

Figura 6: Evolución del CG del fuel.

El CG tiende al óptimo pero sin llegar a alcanzarlo ya que los demás costes lo impiden. Nótese que el CG inicial es nulo, debido a que en la primera iteración ya se ha perturbado la solución inicial.

\subsection{DETERMINACIÓN DE LOS PESOS}

Se trata de un problema con varios objetivos que deben cumplirse, cada uno de ellos con un coste asociado que se debe determinar, ya que la función de coste es la suma ponderada de todos ellos. Por ejemplo, si damos pesos muy grandes al posicionamiento del $\mathrm{CG}$, la solución convergerá a una distribución del fuel cercana al CG óptimo, sin tener en cuenta los otros costes que pueden no alcanzar buenas soluciones (Figura 7).

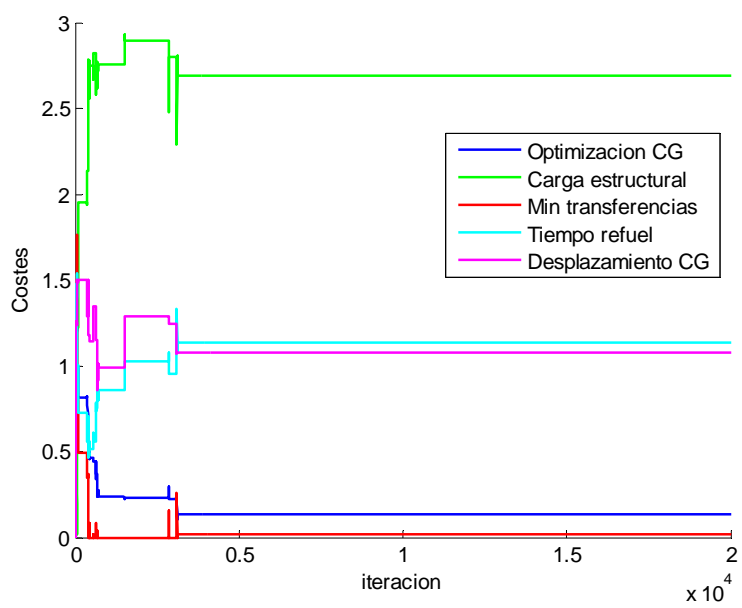

Figura 7: Evolución de los costes asociados al mejor individuo para los pesos $\mathrm{P}=\left[\begin{array}{llllll}10 & 1 & 1 & 1 & 1 & 1\end{array}\right]$.

En la Figura 8 se muestra la evolución del CG del mejor individuo. Efectivamente el CG está muy cerca del CG óptimo, a unos $30 \mathrm{~cm}$ de él.

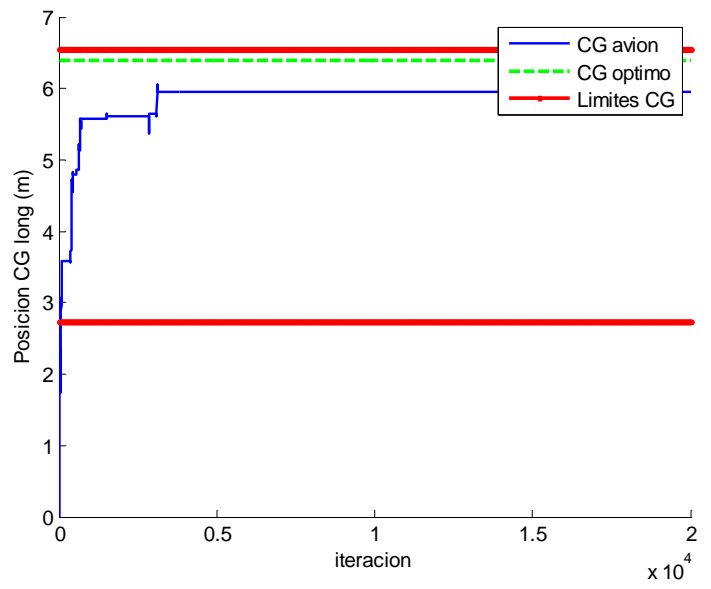

Figura 8: Evolución del CG del fuel completo para

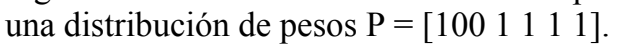

Si en vez de dar tanto peso al posicionamiento del CG se lo damos a otras variables como, por ejemplo, a la minimización del tiempo de transferencia durante el repostaje y a las transferencias durante el vuelo, podemos observar cómo el comportamiento varía bastante respecto al anterior (Figura 9).

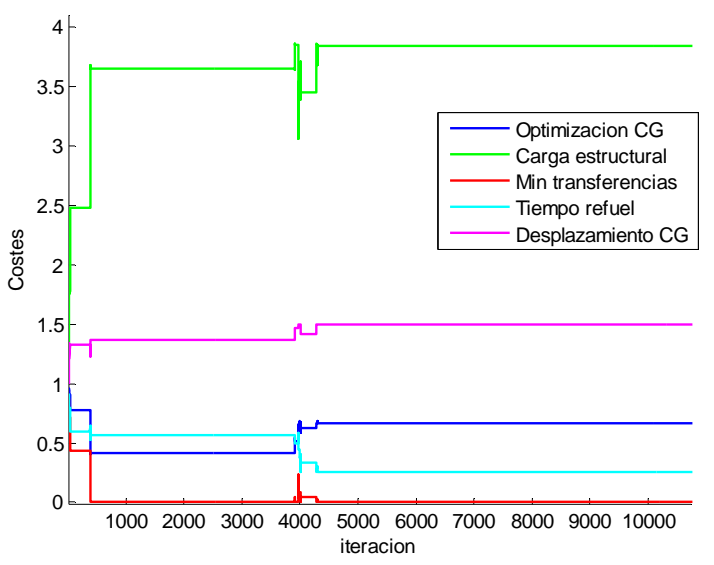

Figura 9: Evolución de los costes asociados al mejor individuo para los pesos $\mathrm{P}=\left[\begin{array}{lllll}1 & 1 & 100 & 100 & 1\end{array}\right]$.

El algoritmos disminuye esos dos factores, y la evolución del CG no tiende a converger al óptimo.

Por otra parte, si igualamos todos los costes, el algoritmo tenderá a minimizar unos costes frente a otros de manera indistinta, con lo que las soluciones del algoritmo serán dispares y no tendrá mucho sentido. Por ello conviene jerarquizar los costes para que haya una tendencia hacia lo que mejora de manera global la solución basándonos en el conocimiento cualitativo del experto.

En nuestro caso se ha premiado ante todo el posicionamiento del CG porque es el principal objetivo de diseño del control. El segundo criterio prioritario ha sido intentar disminuir las transferencias durante el vuelo; esto es, introducir en 
los tanques Inner la masa de fuel más cercana posible a la que previsiblemente consumirán los motores en el vuelo concreto. Esto es importante ya que, ante un fallo total del sistema de transferencia de combustible, los motores no tendrían problema en seguir siendo alimentados. Por ello finalmente se ha optado por el siguiente reparto:

$$
P=\left[\begin{array}{c}
\text { PosicionCG } \\
\text { CargaEstructural } \\
\text { TransferenciasFuel } \\
\text { TiempoRepostaje } \\
\text { DesplazamientoCG }
\end{array}\right]=\left[\begin{array}{c}
100 \\
70 \\
90 \\
60 \\
80
\end{array}\right]
$$

\subsection{COMPARACIÓN CON LA SOLUCIÓN CLÁSICA}

Una cuestión importante es si se mejora la solución que se toma habitualmente en el sector aeronáutico, es decir, el llenado jerárquico de unos tanques concretos hasta cierta cantidad, después otros, y así sucesivamente hasta llegar a introducir toda la masa prevista.

Ejecutamos el algoritmo tomando como solución inicial la clásica, considerando el escenario de 65000 $\mathrm{kg}$ de combustible, carga de pago de $10000 \mathrm{~kg}$ y situándose el $\mathrm{CG}$ del avión para ZFW $0.4 \mathrm{~m}$ por detrás del punto de referencia. Se considera que el $80 \%$ del combustible será consumido por los motores durante el vuelo normal. La evolución de los costes se muestra en la Figura 10.

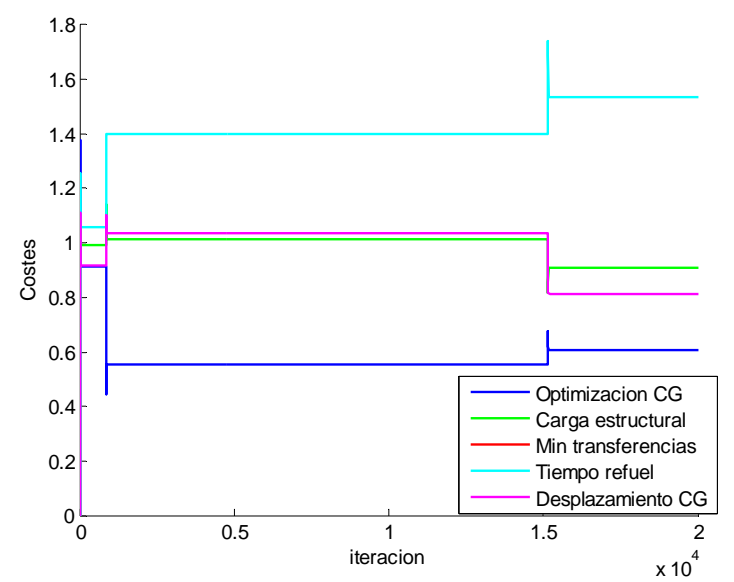

Figura 10: Evolución de los costes asociados al mejor individuo en cada iteración para los pesos.

Observamos como el coste asociado al CG es de los más premiados en el sentido que el algoritmo tiende a disminuirlo rápidamente. $\mathrm{Si}$ presentamos la gráfica de su evolución podemos ver que efectivamente la solución es bastante aceptable, con el CG situado en los márgenes y cerca del óptimo (Figura 11).

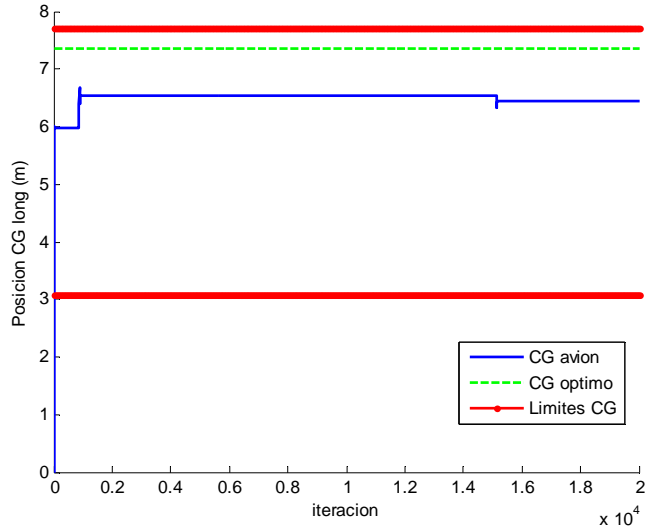

Figura 11: Evolución del CG del fuel para el individuo mejor de cada iteración.

La minimización de transferencias no se observa en la gráfica de los costes pues está con coste " 0 ” todo el rato. Eso es debido a que desde un principio más del $80 \%$ de la masa del fuel se encuentra en los tanques Inner, por lo que el coste de las transferencias es menor. El algoritmo ha premiado las soluciones en las que al menos esa cantidad de fuel permanece en los Inner.

También tiene una clara influencia la minimización del desplazamiento del CG durante el despegue en cada tanque. En las primeras iteraciones el algoritmo encuentra una solución en la que se tiene un CG mejor posicionado a costa de aumentar el desplazamiento de éste durante el despegue. Sin embargo, al evolucionar, se llega a encontrar una solución aún mejor en la que con un leve aumento del coste de la posición del CG se disminuye sustancialmente el coste de su desplazamiento.

El resultado final del algoritmo es el siguiente. Partiendo de una solución inicial de diseño clásica, siguiendo la jerarquía de llenado para $65000 \mathrm{~kg}$ de combustible, que es:

$$
\left.v_{\text {decision }}\right|_{\text {inic }}=[2865,28435,0,28435,2865,2400]
$$

Donde los dos tanques Outer están completos, los Inner casi completos $(56870 \mathrm{~kg}$, es decir, 2 x 28425 kg).), el Central vacío y el Trim con lo restante, el CG del combustible queda a $5.7735 \mathrm{~m}$ por detrás del origen de referencia. El vector de costes sería:

$$
\left.C\right|_{\text {inic }}=\left[\begin{array}{c}
\text { PosicionCG } \\
\text { CargaEstructural } \\
\text { TransferenciasFuel } \\
\text { TiempoRepostaje } \\
\text { DesplazamientoCG }
\end{array}\right]=\left[\begin{array}{c}
158.91 \\
38.55 \\
0 \\
46.61 \\
109.09
\end{array}\right]
$$

La solución final seleccionada por el mecanismo evolutivo es la siguiente: 


$$
\left.v_{\text {decision }}\right|_{\text {final }}=[2852,25681,3519,25681,2852,4414]
$$

Es decir, respecto a la original, se retira fuel de los Outer y de los Inner (51362 kg), sin dejar de mantener prácticamente todo el fuel suficiente para los motores durante el vuelo) y se coloca en el Central y en el Trim. Con esta distribución el CG del combustible queda a 6.4467 metros por detrás del origen de referencia. El vector de costes sería:

$$
\left.C\right|_{\text {final }}=\left[\begin{array}{c}
\text { PosicionCG } \\
\text { CargaEstructural } \\
\text { TransferenciasFuel } \\
\text { TiempoRepostaje } \\
\text { DesplazamientoCG }
\end{array}\right]=\left[\begin{array}{c}
91.5784 \\
34.4197 \\
0.3973 \\
82.9021 \\
94.34
\end{array}\right]
$$

El CG objetivo está a $7.25 \mathrm{~m}$ del origen, por lo que la solución del AG está bastante más cerca del óptimo que la clásica.

\section{CONCLUSIONES Y TRABAJOS FUTUROS}

La optimización heurística aplicada asegura que prácticamente todo el fuel que será consumido por los motores se encuentra en los Inner. Recordamos que esta cantidad de fuel es del $80 \%$ de la original. Es decir, el AG inteligentemente ha sabido retirar toda la cantidad de fuel que sobraba respecto a la que se consumirá por los motores.

Esta cantidad que se retira es utilizada para llenar gran parte del Trim Tank de forma que se retrase el CG. Pero también es utilizada, junto con algunos kg de los Outer, para llevar fuel al Central con dos objetivos: primero, mejorar la homogenización del peso distribuido en las alas (mejora de la carga estructural) y, por otro, mejorar globalmente el desplazamiento del CG durante el despegue.

Por otra parte, la jerarquía de diseño clásica da lugar a una solución bastante buena. Esto es lo esperado, pues es consecuencia del estudio de expertos del tema. El hecho de que no nos separemos mucho de esta solución quiere decir que el diseño de nuestra función objetivo está en la línea del conocimiento experto del problema de repostaje.

Además vale la pena notar que la convergencia del algoritmo a la solución final es buena.

Como trabajos futuros se está abordando el problema de la distribución de combustible durante el vuelo con técnicas inteligentes, en concreto con lógica borrosa. También se propone el plantear una optimización multiobjetivo, con varios costes simultáneos [2].

Por otro lado, sería interesante validar los resultados de la optimización antes de su aplicación real puesto que el componente aleatorio de esta técnica heurística puede hacer que la solución generada no siempre sea mejor que la tradicional.

\section{Agradecimientos}

Este trabajo ha sido parcialmente financiado por la Red Temática Control Inteligente (DPI2015-71320REDT), del Ministerio de Economía y Competitividad).

\section{Referencias}

[1] Airbus, (2017) http://www.airbus.com/aircraftfamilies/passeng eraircraft/a330family/a330-200/

[2] Alonso Zotes, F., Santos, M. (2017) Una revisión de técnicas de optimización heurística para el diseño de trayectorias interplanetarias en misiones espaciales. RIAI, 14 (1) 1-15, 2017

[3] Jimenez, J.F., Girón-Sierra, J.M., Insaurralde, C., Seminario, M., (2007). A simulation of aircraft fuel management system. Simulation Modelling Practice and Theory 15(5) 544-564).

[4] Langton, R., Clark, C., Hewitt, M. and Richards, L., (2009) Aircraft fuel systems. John Wiley \& Sons, Ltd.

[5] Mongeau, M., Bes, C., (2003) Optimization of aircraft container loading. IEEE Trans. on Aerospace \& Electronics Systems 39(1) 140150.

[6] Plaza Alonso, E. (2016) Sistema de control para la distribución de combustible en una aeronave basado en técnicas de la inteligencia artificial, TFM, UNED

[7] Santos, M., de la Cruz, J.M. (2009) Algoritmos genéticos. En: Métodos de Procesamiento Avanzado e Inteligencia Artificial en Sistemas Sensores y Biosensores, Reverté, cap. 15, 321333.

[8] Turgut, E.T., Rosen, M.A., (2012) Relationship between fuel consumption and altitude for commercial aircraft during descent: preliminary assessment with a genetic algorithm. Aerospace Science and Technology, 17(1) 65-73. 